\title{
Directional lithium hydride Compton profiles of $0.1 \%$ statistical accuracy
}

\section{Huotari, Simo}

2009

Huotari , S , Boldrini , B , Honkimäki , V , Suortti , P \& Weyrich , W 2009 , ' Directional lithium hydride Compton profiles of $0.1 \%$ statistical accuracy ' , Journal of Synchrotron Radiation , vol. 16 , no. 5 , pp. 672-682 . https://doi.org/10.1107/S0909049509029112

http://hdl.handle.net/10138/23559

https://doi.org/10.1107/S0909049509029112

publishedVersion

Downloaded from Helda, University of Helsinki institutional repository.

This is an electronic reprint of the original article.

This reprint may differ from the original in pagination and typographic detail.

Please cite the original version. 
Journal of

\title{
Synchrotron
}

\section{Radiation}

ISSN 0909-0495

Editors: G. Ice, Å. Kvick and T. Ohta

\section{Directional lithium hydride Compton profiles of $0.1 \%$ statistical accuracy}

\author{
Simo Huotari, Barbara Boldrini, Veijo Honkimäki, Pekka Suortti and Wolf \\ Weyrich
}

J. Synchrotron Rad. (2009). 16, 672-682

Copyright (C) International Union of Crystallography

Author(s) of this paper may load this reprint on their own web site or institutional repository provided that this cover page is retained. Republication of this article or its storage in electronic databases other than as specified above is not permitted without prior permission in writing from the IUCr.

For further information see http://journals.iucr.org/services/authorrights.html

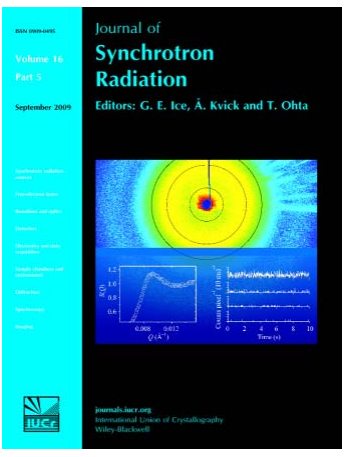

Synchrotron radiation research is rapidly expanding with many new sources of radiation being created globally. Synchrotron radiation plays a leading role in pure science and in emerging technologies. The Journal of Synchrotron Radiation provides comprehensive coverage of the entire field of synchrotron radiation research including instrumentation, theory, computing and scientific applications in areas such as biology, nanoscience and materials science. Rapid publication ensures an up-to-date information resource for scientists and engineers in the field.

\section{Crystallography Journals Online is available from journals.iucr.org}


Journal of

Synchrotron

Radiation

ISSN 0909-0495

Received 5 May 2009

Accepted 22 July 2009

\section{Directional lithium hydride Compton profiles of $0.1 \%$ statistical accuracy}

\author{
Simo Huotari, ${ }^{a, b *}$ Barbara Boldrini, ${ }^{\mathrm{c}}$ Veijo Honkimäki, ${ }^{\mathrm{a}}$ Pekka Suortti ${ }^{\mathrm{b}}$ and \\ Wolf Weyrich ${ }^{\mathrm{c}}$
}

${ }^{\mathbf{a}}$ European Synchrotron Radiation Facility, BP 220, F-38043 Grenoble, France, ${ }^{\mathbf{b}}$ Division of Materials Physics, Department of Physis, Fl-00014 University of Helsinki, Finland, and ' ${ }^{\text {C University }}$ of Konstanz, Department of Chemistry, Fach 721, D-78457 Konstanz, Germany.

E-mail: simo.huotari@esrf.eu

\begin{abstract}
The present status and accuracy of determining the electron momentum density from experimental Compton profiles is reviewed. The new spectrometers operating at third-generation synchrotron radiation sources have made possible measurements with $0.1 \%$ statistical accuracy at the Compton peak. A comparable accuracy of the Compton profiles is achieved only after careful corrections for departures from the impulse approximation, effects of multiple scattering, and variations in the analyser response function. Detailed descriptions are given of the correction procedures applied to the data collected by the Johann-type scanning spectrometer that is one of the Compton spectrometers in use at the ESRF. Special attention is paid to the calculation and correction of the glitches that are caused by extra reflections of the analyser crystal. The Fourier transform of the Compton profile, the reciprocal form factor, is calculated, and its use in data treatment and presentation is discussed.
\end{abstract}

Keywords: lithium hydride; electron momentum density; directional Compton profiles; reciprocal form factor.
(C) 2009 International Union of Crystallography Printed in Singapore - all rights reserved et al., 1992; Suortti et al., 1999, 2001; Hiraoka et al., 2001, $2005 b$ ). In all designs, bent crystal analysers in focusing geometry are used. At the high-energy beamlines of the thirdgeneration facilities the monochromators and detectors are also integrated and optimized in the construction. There are two generic analyser constructions: dispersing crystals in stationary transmission (Cauchois) geometry and monochromatizing crystals in scanning reflection (Bragg) or transmission (Laue) geometries (DuMond, 1947). In the first case there are no moving parts, and the analyser disperses the scattered radiation in such a way that there is a one-to-one correspondence between energy and position at the one- or two-dimensional detector. In the second case the sample and the analyser are on the Rowland circle, and by synchronized motions one energy at a time is reflected to the detector, which is on the Rowland circle in the Bragg case, or looks into the virtual focus in the Laue case. In the case of the dispersive spectrometer the energy resolution is determined mostly by the detector resolution, while in the scanning spectrometer the resolution is determined by focusing of the incident beam on the sample, and the sample size. Both types of spectrometers have their advantages and disadvantages, but under optimized conditions comparable resolutions and count rates are achieved.

The performance of the earlier focusing spectrometers working at synchrotron radiation laboratories or with X-ray 
tubes as the sources of radiation was limited by low flux of monochromatic incident radiation, low photon energy and inefficient detectors. The statistical accuracy remained on the $1 \%$ level, the momentum resolution was about $0.15 p_{0}$ at best, ${ }^{1}$ and the conditions of the impulse approximation (IA) were not always fulfilled. The new spectrometers work in the energy range $30 \mathrm{keV}$ to $115 \mathrm{keV}$, and a momentum resolution of $0.05 p_{0}$ has been achieved; even $0.02 p_{0}$ at lowest energies (Huotari et al., 2000, 2007). Owing to the increased energy the effects of absorption are reduced, and the deviations from the IA are negligible or they can be calculated. At the Compton peak the count rate is typically $10000 \mathrm{~s}^{-1} p_{0}{ }^{-1}$, and more than ten million counts over the whole Compton profile can be collected in one shift of $8 \mathrm{~h}$.

The experimental advances in Compton scattering have opened totally new avenues for research. Fermi surface mapping can be carried out by reconstructing the electron momentum density from a sufficient number of the directional Compton profiles (DCP) (Schülke et al., 1996; Tanaka et al., 2001; Hiraoka et al., 2005a), and the nature of chemical bonding can be studied in detail (Bräuchler et al., 1989; Gillet et al., 1995). The momentum resolution has become comparable with those achieved by angular correlation of annihilation radiation (ACAR) and inelastic electron scattering (e,2e), but Compton scattering is not hampered by sample defects or strong multiple scattering. In ACAR the positron wavefunction may weight selectively the electron momentum density and may even render some parts of it invisible (Shukla et al., 1999).

High-resolution measurements with good statistical accuracy indicated that in contrast to the generally accepted picture of the Compton scattering process the final-state effects are important (Sternemann et al., 2000; Soininen et al., 2001) and limit the momentum resolution at low incident photon energies (below $30 \mathrm{keV}$ ). This explains partly the observed smearing of the edge of the Compton profile at the Fermi energy (Sakurai et al., 1995; Schülke et al., 1996; Huotari et al., 2000). On the other hand, it was verified that thermal motion does not smear the Compton profile (Sternemann et al., 2001; Huotari et al., 2002), contrary to some theoretical predictions (Dugdale \& Jarlborg, 1998). The advances on the experimental side have focused attention on some fundamental questions and have stimulated theoretical work. In short, the dialogue continues now on a higher level of sophistication and understanding.

Much of the earlier work on single-crystal samples concentrated on observing directional differences between the Compton profiles. In such comparisons most experimental errors or corrections to the profiles cancel out. Important results were obtained even in experiments where the momentum resolution was of the order of $0.5 p_{0}$, or where the statistical accuracy was low (Pattison \& Weyrich, 1979; Asthalter et al., 1992; Asthalter \& Weyrich, 1993; Loupias \& Mergy, 1980). Some of the traditional data treatment methods

${ }^{1} p_{0}$ is the atomic linear-momentum unit $\hbar / a_{0}=1$ DuMond (in analogy to $a_{0}=$ 1 Bohr for the atomic unit of length). were carried over to recent studies, like the practice of taking the average of the low-energy and high-energy sides of the profile, or smoothing intensity variations. Unfortunately, errors or interesting details in data may remain hidden after such data treatment. New approaches are needed for a full use of the information in the present-day experiments.

The improvements in momentum resolution, statistical accuracy and the use of higher incident photon energies have been accompanied by developments in extracting the actual Compton profiles from the recorded intensity of scattering, and in further data analysis. Most of the development work has been performed at the synchrotron radiation laboratories in collaboration with groups using the new spectrometers (Suortti et al., 1999; Hiraoka et al., 2001). The solutions depend on the type of the spectrometer, but the problems are common. These include at least corrections for the departure from the conditions of the IA, correction for multiple scattering, and counting for the effects of the energy-dependent response function of the analyser. The last one includes the variation of the bent-crystal reflectivity and the change of the energy resolution across the Compton profile. In particular, the reflectivity of the analyser deserves careful analysis, because many reflections may take place simultaneously and give rise to so-called glitches in the recorded spectrum.

The details of data treatment are seldom reported at length. The present work is a part of a wide-scope project, where the ultimate aim is to derive density matrices from Compton profiles and structure factors, i.e. to calculate an experimental electron wavefunction in a crystalline solid. For this goal, fully corrected Compton profiles are needed in many crystallographic directions, and the statistical noise must be $\leq 0.1 \%$ of the peak value of the Compton profile. It became evident in the course of the project that no short cuts could be taken, but all the corrections had to be evaluated and applied individually to ensure that theory is met at a sufficiently high level of accuracy. For instance, the kinetic energy per formula unit can be calculated from the second moment of the Compton profile (Nyanda \& Williams, 1979; Weyrich, 1979; see also Nygård et al., 2007), but the result is extremely sensitive to small errors in the profile (Weyrich, 1979). In the following, we report in some detail experimental methods and data treatment that are specific to the scanning spectrometer being used at High Energy Beamline ID15B of the European Synchrotron Radiation Facility (ESRF). However, many of the results are general and can be applied to data recorded by other spectrometers.

\section{Compton profile and reciprocal form factor}

The aim of the present work is to provide procedures for evaluating fully corrected Compton profiles from the recorded energy spectrum of the scattered radiation. Only a short account of the basics of Compton scattering is given in the following. The data can be analysed using the Compton profile or its Fourier transform, the reciprocal form factor, so that the definitions and relations of these are given below. This combined analysis has some practical advantages, and the 
reciprocal form factor provides a link between position and momentum space distributions.

The electron position density (charge density) $\rho(\mathbf{r})$ is the Fourier transform of the elastic scattering amplitude, $F(\mathbf{k})$, which is given in units of the electron scattering length (classical electron radius) $r_{\mathrm{e}}=e^{2} / m c^{2}=2.818 \times 10^{-5} \AA$. Here $\mathbf{r}$ and $\mathbf{k}$ are the position coordinate and the scattering vector, respectively. The values of $F(\mathbf{k})$ are obtained at the reciprocal lattice points $\mathbf{k}_{h k l}$ from a diffraction experiment, if the phase of $F(\mathbf{k})$ can be solved, and $\rho(\mathbf{r})$ is then calculated from the Fourier series.

The counterpart of the electron position density $\rho(\mathbf{r})$ is the electron momentum density (EMD) $\varpi(\mathbf{p})$, and it is obtained from the energy loss of the probing high-energy photon in $(\mathrm{X}, \mathrm{eX})$ scattering or the energy loss of the electron in $(\mathrm{e}, 2 \mathrm{e})$ scattering, when the recoil electron is observed in coincidence. In the conventional Compton scattering only the scattered photon is detected, and its energy is analysed. The motion of the target electrons causes Doppler broadening in the energy of the scattered photons, and the observed spectrum is called the (uncorrected) Compton profile in the energy scale $\hbar \omega_{2}$ or simply the Compton band $I\left(\hbar \omega_{2}\right)$, the subscript 2 indicating the scattered photon. The (corrected) Compton profile $J$ on the momentum scale is the one-dimensional projection of the EMD on the scattering vector $\mathbf{k}=\mathbf{k}_{2}-\mathbf{k}_{1}$, which is usually chosen as the $z$-direction of the coordinate system of the experimental set-up. In that coordinate system the relationship between $\varpi(\mathbf{p})$ and $J\left(p_{z}\right)$ is

$$
J\left(p_{z}\right)=\int_{-\infty}^{\infty} \int_{-\infty}^{\infty} \varpi(\mathbf{p}) \mathrm{d} p_{x} \mathrm{~d} p_{y},
$$

an area integration over the $\left(\mathbf{p}_{x}, \mathbf{p}_{y}\right)$-plane, to which the $\left(\mathbf{p}_{z} \|\right.$ $\mathbf{k}$ ) direction is perpendicular. In the sample coordinate system with variable orientations of the sample relative to the scattering vector $\mathbf{k}$ and therefore variable orientations of the scattering vector with respect to the sample, the relationship becomes the volume integral

$$
J(\mathbf{q})=\int_{V_{\mathbf{p}}} \varpi(\mathbf{p}) \cdot \delta\left(\frac{\mathbf{p} \cdot \mathbf{q}}{q}-q\right) \mathrm{d} \tau_{\mathbf{p}} .
$$

The electron momentum vector $\mathbf{q}$ is independent of the choice of any coordinate system and points in the direction of the $\mathbf{k}$ vector of the particular measurement. With infinitely many such measurements with different orientations of $\mathbf{k}$ the threedimensional Compton-profile function $J(\mathbf{q})$ is obtained as a continuous function in momentum space, whose only peculiarities are that it is a probability density distribution of the modulus $q=|\mathbf{q}|$ and that it can be multivalued at $q=0$ (Janis $e t$ $a l ., 1978)$, written as $J\left(0, \vartheta_{q}, \varphi_{q}\right)=J(0)$ (Weyrich, 1978). The two expressions (1) and (2) are naturally equivalent, but seen from different coordinate systems. Thinking in terms of the latter expression is useful when the sample coordinate system is preferred to be kept fixed and measurements to be performed in arbitrary directions of $\mathbf{q}$.
The EMD is the sum of one-electron momentum densities over the occupied natural spin orbitals $\tilde{\psi}_{j}(\mathbf{p})$ in momentum space,

$$
\varpi(\mathbf{p})=\sum_{j} n_{j} \varpi_{j}(\mathbf{p})=\sum_{j} n_{j} \tilde{\psi}_{j}(\mathbf{p}) \tilde{\psi}_{j}^{*}(\mathbf{p}),
$$

where $0 \leq n_{j} \leq 1$ is the occupation number, and $\tilde{\psi}_{j}(\mathbf{p})$ is the three-dimensional Fourier transform of the one-electron wavefunction $\psi_{j}(\mathbf{r})$ in position space. Correspondingly, the electron position density is written as

$$
\rho(\mathbf{r})=\sum_{j} n_{j} \rho_{j}(\mathbf{r})=\sum_{j} n_{j} \psi_{j}(\mathbf{r}) \psi_{j}^{*}(\mathbf{r}) .
$$

By the definition of $\varpi(\mathbf{p})$, for any direction of $\mathbf{q}$,

$$
\int_{-\infty}^{\infty} J(\mathbf{q}) \mathrm{d} q=N
$$

where now $N$ is the number of electrons in the scattering unit (atom, molecule, asymmetric unit, formula unit).

$\rho(\mathbf{r})$ and $\varpi(\mathbf{p})$ are united in the quantum-mechanical counterpart of phase space, the one-electron density matrix $(\mathrm{ODM})$. In its pure position-space representation, $\rho(\mathbf{r})$ is the diagonal of the ODM, whereas $\varpi(\mathbf{p})$ is represented by the socalled reciprocal form factor $B(\mathbf{s})$, which is the Fourier transform of the EMD,

$$
B(\mathbf{s})=\int_{V_{\mathbf{p}}} \varpi(\mathbf{p}) \exp (-i \mathbf{s} \cdot \mathbf{p}) \mathrm{d} \tau_{\mathbf{p}} .
$$

When the convolution theorem of the Fourier transformation is applied, and the EMD is decomposed into the sum of orbital contributions,

$$
\begin{aligned}
B(\mathbf{s}) & =\sum_{j} n_{j} \int_{V_{\mathbf{r}}} \psi_{j}(\mathbf{r}) \psi_{j}^{*}(\mathbf{r}+\mathbf{s}) \mathrm{d} \tau_{\mathbf{r}} \\
& =\int_{V_{\mathbf{r}}} \gamma(\mathbf{r}, \mathbf{r}+\mathbf{s}) \mathrm{d} \tau_{\mathbf{r}} .
\end{aligned}
$$

Accordingly, $B(\mathbf{s})$ is the sum of autocorrelation functions of the position-space orbitals or, more precisely and more generally, $B(\mathbf{s})$ is the projection of the off-diagonal parts of the ODM onto the subspace $\{\mathbf{s}\}$ perpendicular to the diagonal of the matrix (Weyrich, 1978, 1996; Asthalter \& Weyrich, 1997).

It is seen from (6) that in the direction $s_{z} \| p_{z}$ (in three dimensions $\mathbf{s} \| \mathbf{q})$ the reciprocal form factor is the onedimensional Fourier transform of the (directional) Compton profile,

$$
B\left(s_{z}\right)=\int_{-\infty}^{\infty} J\left(p_{z}\right) \exp \left(-i s_{z} p_{z}\right) \mathrm{d} p_{z}
$$

It is customary to use atomic units, i.e. to express the length $s_{z}$ in multiples of the Bohr radius $a_{0}=0.529177 \times 10^{-10} \mathrm{~m}=$ 1 Bohr and the momentum $p_{z}$ in multiples of $p_{0}=h / 2 \pi a_{0}=$ $1.99285 \times 10^{-24} \mathrm{~kg} \mathrm{~m}^{-1}=1$ DuMond. The frequent omission of the various atomic units in relationships is equivalent to 
setting $h=h / 2 \pi=1, m_{\mathrm{e}}=1$ and $c=137.036$ (inverse of the finestructure constant $\alpha$ ). The analysis of the experimental data is carried out alternatively in terms of $J\left(p_{z}\right)$ or $B\left(s_{z}\right)$, which has certain practical advantages, and the results can be used for subsequent reconstruction of the three-dimensional momentum and position space densities, and for comparisons with theory.

\section{Experiment}

The goal of the experiment was to produce a data set of directional Compton profiles (DCPs) with sufficient statistical accuracy and momentum resolution for a detailed comparison with the most sophisticated theoretical calculations. At the same time, the data should be sufficiently extensive for mapping an irreducible volume of reciprocal space and reconstruction of the three-dimensional EMD. Accordingly, an experimental strategy was developed to allow the recording of 15 DCPs in five days, with about $10^{6}$ counts per $0.1 p_{0}$ at the Compton peak. The measurements were carried out using the Johann-type scanning spectrometer at beamline ID15B of the ESRF. One of the advantages of scanning spectrometers is that counting statistics can be optimized at different count rates. We describe in detail the experimental conditions and reduction of the recorded data to Compton profiles, because the level of accuracy needed for comparison with theory can be achieved only when all the factors affecting the measurement are fully understood.

The construction and performance of the first scanning spectrometer at ID15B have been described in detail (Suortti et al., 1999). Radiation from an asymmetric multipole wiggler is monochromatized and focused on the sample by a horizontally bent $\mathrm{Si}$ crystal with a 5:1 demagnification ratio. In the present work the (311) reflection was used to produce $57.00 \mathrm{keV}$ radiation with about $30 \mathrm{eV}$ bandwidth. A slit of $0.3 \mathrm{~mm}$ (horizontal) $\times 5 \mathrm{~mm}$ (vertical) was placed at the exit of the beam tunnel, and the flux was monitored by a Si pin diode. Secondary monitoring was performed by recording the intensity of total scattered radiation from the sample with a Ge detector placed at a $140^{\circ}$ scattering angle. The scan routine included frequent tuning of the monochromator for a maximum flux. The advantage of the horizontally focused beam is that small needle-like samples can be used to minimize multiple scattering. The spectrometer works in the horizontal Rowland-circle geometry, where the scan motions maintain the sample, analyser and detector on the focusing circle. The analyser was a cylindrically bent Johann-type Ge (440) crystal. Owing to the focusing geometry, a narrow slit ( $2 \mathrm{~mm}$ ) could be used in front of the detector, which made the background very low, only about $0.1-0.2 \%$ of the Compton peak intensity. The vertical opening angle of the receiving slit, as seen from the sample position, is about $40 \mathrm{mrad}\left(= \pm 1.2^{\circ}\right)$ at the position of the Compton peak.

The sample geometry (Fig. 1) was similar to that used in the experiment on Be (Huotari et al., 2000). Two single-crystalline $\mathrm{LiH}$ samples, about $15 \mathrm{~mm}$ long with a rectangular cross section, where the edges varied from $1.0 \mathrm{~mm}$ to $1.6 \mathrm{~mm}$, were

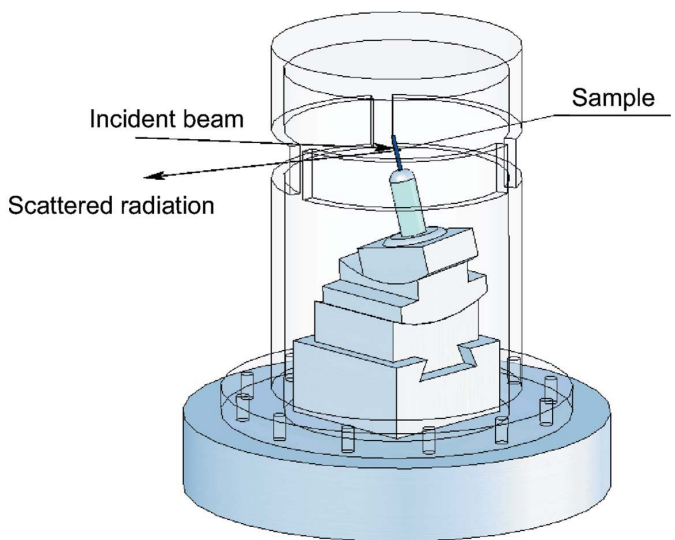

Figure 1

Sample environment in the present experiment. The sample is a rectangular stick kept in a vacuum chamber during the measurements.

cut by cleaving and with a diamond saw from a crystal boule. The long axes of the crystals were in the $\langle 100\rangle$ and $\langle 110\rangle$ directions, respectively. $\mathrm{LiH}$ is very sensitive to moisture, air and carbon dioxide. Therefore, the crystal boule was stored in a sealed bag filled with paraffin oil, and cutting was carried out in a glove-box under dry argon atmosphere. For transportation the samples were sealed in ampoules with paraffin oil, which was removed before the experiment with pentanehexane mixture. The samples were mounted on a goniometer head inside a glove box with an Ar atmosphere, and transported to the beamline in a vacuum chamber that had mylar windows for the incident, transmitted and scattered beams. During the experiment the chamber was continuously kept evacuated to a pressure of $<1$ mbar. The samples were examined after the experiment under a microscope. No visible surface degradation was observed.

The samples were oriented in such a way that the scattering vector directions corresponded to an approximately uniform grid in the irreducible 1/48 area of the unit sphere (see Fig. 2). The rationale for that strategy has been not to introduce a directional bias for the spherically averaged $J(q)$ and the later

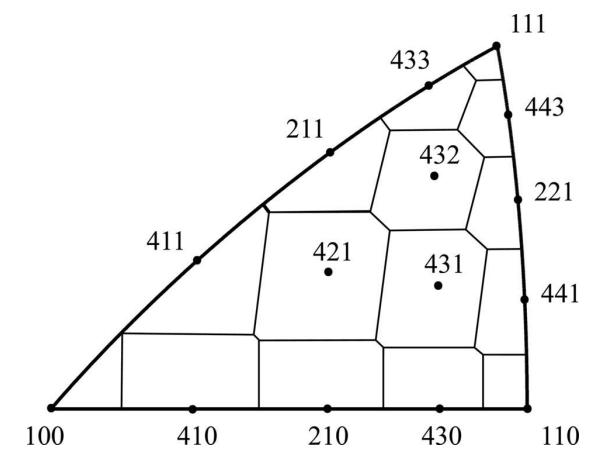

Figure 2

The irreducible $1 / 48$ sector of the unit sphere showing the crystallographic directions for which the Compton profiles were acquired. The polygons around the directional points are the Voronoi cells whose area (= solid angle) indicates the weight of that direction for the twodimensional representation of the directional dependence of $\varpi(\mathbf{p}), J(\mathbf{q})$, $B(\mathbf{s})$, and other quantities not discretized by the Fourier sum over the three-dimensional positional crystal lattice points. Those weights should therefore not be confused with the crystallographic multiplicities of structure factors even for crystallographically cubic systems. 
reconstruction of the three-dimensional electron momentum density $\varpi(\mathbf{p})$. Fortunately, in the cubic structure of $\mathrm{LiH}$ that choice almost coincides with the goal to obtain data along the directions of the interionic vectors $\mathbf{R}_{m n}$ for studying the interionic interactions with the highest sensitivity. Therefore we have chosen the directions of $\mathbf{R}_{m n}=(1 / 2) \mathbf{R}_{u v w}$ with $u=4$, $w \leq v \leq 4,0 \leq w \leq 4$, thus covering all interionic interactions within the cube of $4 \times 4 \times 4=64$ unit cells. The resulting 15 irreducible directions are [400], [410], [420], [430], [440], [411], [421], [431], [441], [422], [432], [442], [433], [443] and [444]. Interionic distances $\mathbf{R}_{x y z}$ with integer $2 x, 2 y, 2 z$ that are a fraction or a multiple of any $\mathbf{R}_{u v w}$ are automatically included.

The vectors to the directions along the line from [110] through [111] to [001] are perpendicular to [110], so that nine points of the 15-point grid could be reached by rotating the $\langle 110\rangle$ crystal about its axis, which was perpendicular to the scattering plane. In the same way, the points between [100] and [110] were reached by rotating the $\langle 100\rangle$ crystal about its axis. The three remaining points inside the triangle were reached by small tilts of either crystal. The tilt needed for reaching [421] from $[1,1 / 2,0]$, for example, is $12.60^{\circ}$, and the angle to $[1,3 / 4,1 / 2]$ from $[1,1,1 / 2]$ is $7.96^{\circ}$. Owing to the almost backscattering geometry with $\Theta=172.55^{\circ}$, to a very good approximation the tilt is in the direction of the incident beam. The projected length of the scattering volume increases by $h \tan \beta$, where $h$ is the height of the beam and $\beta$ is the tilt angle. The effect on the energy resolution of the spectrometer will be briefly discussed further below. The sample was aligned to a reference direction (either [100] or [110]) along the incident beam by bringing the 002 and 004 reflections to the horizontal plane, using a fluorescent screen as the detector. The accuracy of the alignment was better than $0.1^{\circ}$.

The resolution function of the scanning spectrometer depends on the incident beam distributions, the illuminated volume of the sample and the reflectivity of the analyser. Usually the elastically scattered radiation provides a reference line, which can be used for calculation of the energy and momentum resolution at the Compton peak. In addition, the Compton profile is broadened owing to variation of the scattering angle, but this effect was negligible in the present case. Elastic scattering of $57 \mathrm{keV}$ radiation from $\mathrm{LiH}$ was very weak, and therefore an $\mathrm{Al}$ sample of the same size as the $\mathrm{LiH}$ samples was used for calibration. The effective thickness could be varied from $1.2 \mathrm{~mm}$ to $1.95 \mathrm{~mm}$ by rotating the sample. This range covers the effective thickness of the $\mathrm{LiH}$ samples in different orientations, including the cases where the sample was tilted. There was a small systematic increase in the width of the elastic line with increasing sample thickness, and the shape of the line was symmetric and Gaussian. The FWHM of the resolution function in the momentum scale varied from $0.20 p_{0}$ to $0.22 p_{0}$ at the Compton peak. Individual resolution functions were assigned to the Compton profiles, corresponding to different crystallographic directions. A momentum resolution of $0.15 p_{0}$ could have been reached by using a narrower collimating slit $(0.2 \mathrm{~mm})$, but in the present case the highest resolution was not needed, and the intensity was maximized instead.
The beam reflected by the analyser crystal was recorded by a scintillation detector. The energy resolution of the photon counting chain is about $10 \mathrm{keV}$ at $50 \mathrm{keV}$, so that parasitic components such as the detector escape peak and Ge fluorescence from the analyser crystal can be separated (Suortti et al., 1999). The highest count rates at the Compton peak were about $7000 \mathrm{~s}^{-1}$, which required a dead-time correction. The detector time constant $\tau=1.4 \mu$ s was much larger than the inter-bunch distance of the ESRF storage ring in uniform fill, so that a steady-state radiation source could be assumed (Laundy \& Collins, 2003). Each profile was measured many times using an automatic scan routine where statistics were optimized by varying the counting time from $1 \mathrm{~s}$ per point at far tails to $44 \mathrm{~s}$ per point at the Compton peak area. These choices of counting times yield an almost constant statistical accuracy across the profile (Weyrich, 2010).

One scan with interruptions for monochromator fine-tuning lasted about $1 \mathrm{~h}$, and the scans were repeated four to eight times. A common scale for the profiles was obtained by dividing the sum of scans at each point of measurement by the respective cumulated monitor count. The background owing to the detector dark current and the background from the hutch environment when the sample was removed were measured and subtracted. There was a small residual background, presumably owing to Compton scattering from the analyser crystal, which decreases as $Q^{-2}$, where $Q$ is the distance between the analyser and the detector. This component was estimated to be 1 to 2 counts per second under the profile, i.e. less than $0.1 \%$ of the intensity at the Compton peak, and a parabola was fitted at the far tails of the Compton profile, where the intensity should be zero.

Since the quasielastic line had a very weak intensity, it could not be used for an angle-scale calibration of the spectrometer as is done usually. Because of this, the calibration was carried out with a very high accuracy using a glitch of the analyser crystal located at the Bragg angle $\theta_{\mathrm{B}}=7.49449^{\circ}$, as will be described in $\$ 4.2$. The $\theta_{\mathrm{B}}$ scale was converted to energy $E$ of the scattered photon via Bragg's law. As the integrated reflectivity of an analyser crystal varies as a function of photon energy, the spectra were corrected for this variation based on calculations by Erola et al. (1990). The spectra were corrected for other energy-dependent factors, namely the sample selfabsorption as well as the absorption in air between the sample and the detector, and for the quantum efficiency of the $\mathrm{NaI}$ scintillation detector. Finally, the corrected profiles were converted to DCPs in the momentum scale $q$ using relativistic formulae for the $q$ versus $E$ relation, and the corresponding cross section (Holm, 1988).

\section{Corrections to Compton profiles}

\subsection{Impulse approximation, multiple scattering, resolution function}

The Compton profile is symmetric because of the inversion symmetry of the electron momentum density in the absence of net magnetic fields. The experimentally determined Compton 
profiles typically have deviations from this rule, even after all the corrections and conversions discussed above. Reasons for this are multiple scattering, change of the resolution function across the profile, the failure of the IA, and final-state effects. The essence of the IA is that in order to properly probe the ground-state momentum density the scattering process must be instantaneous, i.e. the energy and momentum transfers must be very large compared with the binding energy and characteristic momentum of the electron (Eisenberger \& Platzman, 1970). Final-state effects are related to the IA and can become important if the scattering electron is not transferred in the far continuum (Sternemann et al., 2001). The reasons for the profile asymmetry have been studied in detail (Huotari et al., 2001). The final-state effects can be ruled out in the present case owing to the high-energy photons involved in the study. The small deviation from the IA is due to the two is electrons of $\mathrm{Li}^{+}$, and it was calculated using hydrogenic wavefunctions (Holm \& Ribberfors, 1989). The more complicated quasi-self-consistent-field calculation of the coreelectron Compton profiles gives essentially the same result (Issolah et al., 1991; Huotari et al., 2001). Multiple scattering is small in the present geometry, where the irradiated sample volume is comparable with the total sample volume. A correction was calculated using an efficient Monte Carlo code, where the polarization of the photons is included throughout the calculation using Stokes parameters (Fajardo et al., 1998). The peak of the multiple-scattering contribution is at a slightly lower energy than the peak of single scattering. The total amount of multiple scattering was $1.7 \%$ at most, and the maximum asymmetry owing to multiple scattering was $0.1 \%$ of $J(0)$. The width of the resolution function is approximately proportional to $E^{3}$ at the Compton peak, which broadens the profile slightly more on the high-energy side than on the lowenergy side (Suortti et al., 1999). The effect is easily calculated analytically when the Compton profile is approximated by a sum of Gaussians. The maximum change in $J(q)$ at $q= \pm 1 p_{0}$ was $\pm 0.02 \%$ of $J(0)$, which is negligible. More important are the variations in the thickness and shape of the irradiated sample as seen by the analyser (Boldrini \& Weyrich, 2010). For each orientation, this effect has been corrected via its reciprocal form factor to the common value $\Delta q_{\mathrm{FWHM}}=$ $0.210 p_{0}$, which is a prerequisite for correct spherical averaging and for the future reconstruction of the three-dimensional electron momentum density $\varpi(\mathbf{p})$. The results for the calculated effects of the IA departure, multiple scattering and the varying resolution function are collected in Fig. 3. The part of the corrections to $J(q)$ that is antisymmetric about $q=0$ justifies the common practice of taking the average of the Compton profile at $-q$ and $+q$.

\subsection{Quantum efficiency function of the analyser}

After the above corrections the profiles should be perfectly symmetric. However, the response or quantum efficiency function of the crystal analyser is not exactly a smooth function of the angle of incidence, but there are small dips and sometimes bumps. The (440) planes of the analyser crystal are

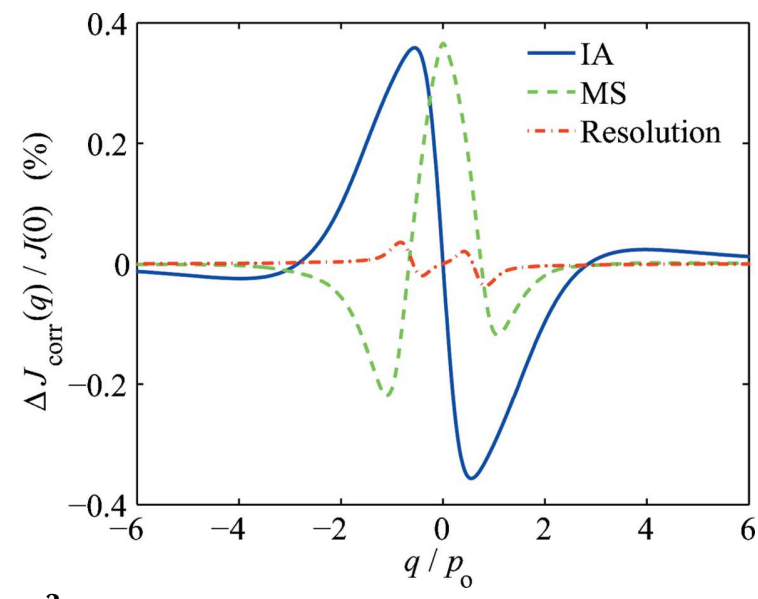

Figure 3

Corrections for deviation from the IA, for multiple scattering (MS) and for the varying resolution function.

not the only ones that reflect the beam scattered by the sample. When the analyser crystal is rotated about the vertical [110] axis the reciprocal lattice point (relp) (440) stays on the Ewald sphere, but many other points pass through the sphere, i.e. they fulfil the reflection condition at certain angles. The chances of reflection are multiplied when the beam has axial (vertical) divergence.

The beam from such an extra reflection may reach the narrow but vertically extended receiving slit and contribute to detectable intensity, if the relp of the reflection lies in the vertical plane that is spanned by the nominal relp (440) and the vertical rotation axis, and thus fulfils the horizontal focusing condition at least approximately. This has been observed both for an off-focusing-plane reflection \{(551) with [001] as the rotation axis $\}$ and for parasitic scattering excited by incident beam harmonics and reflected by other $(h h 0)$ of the analyser (Manninen et al., 1996; Suortti et al., 1999). Both types of unwanted irregular contributions to the measured spectrum are avoided in the present set-up by choosing the (311) reflection of the $\mathrm{Si}$ monochromator (overtones forbidden or very weak), and by choosing [1ํㅣㄹ as the rotation axis of the analyser crystal (Suortti et al., 1999).

More intricate are the effects of multiple diffraction in the analyser crystal, which occur whenever not only (000) and the observed $(h k l)$ [here (440)] but also one or more additional relps are on the Ewald sphere simultaneously. Depending on the moduli and the relative phases of the structure factors of all the relps, the observed reflection can gain (Umweganregung) or lose (Aufhellung) intensity.

The occurrence of multiple diffraction is primarily a geometrical question. Under the ideal Rowland-circle condition in the present case, the direction of the Compton-scattered photon with wavelength $\lambda$ has to lie in the plane that contains (000) and (440) and that stays perpendicular to the rotation axis [1 10 ] [plane of all $(h h l)]$. The centre of the Ewald sphere is located on the plane that is perpendicular to the reciprocal lattice vector from (000) to (440) and bisects it at (220). In the units of the reciprocal unit cell edge $a^{*}$, the radius of the sphere is $r_{\mathrm{E}}^{*}=\left[8+\left(z_{\mathrm{E}}^{*}\right)^{2}\right]^{1 / 2}=8^{1 / 2} / \sin \theta_{\mathrm{B}}=\left(a^{*} \lambda\right)^{-1}$. Here 
$z_{\mathrm{E}}^{*}$ is the distance from (220) to the centre of the Ewald sphere, i.e. $z_{\mathrm{E}}^{*}=8^{1 / 2} / \tan \theta_{\mathrm{B}}$. The centre of the Ewald sphere is located at $\left(2+z_{\mathrm{E}}^{*} \sin \psi ; 2-z_{\mathrm{E}}^{*} \sin \psi ; z_{\mathrm{E}}^{*} \cos \psi\right)$, where $\psi$ is the angle between the line from (220) to the centre and the plane [110]. Here $\psi$ is positive with clockwise rotation about the vector (440). For $\psi=0$, the Bragg angle $\theta_{\mathrm{B}}$ or equivalently the wavelength $\lambda$ is the only remaining variable parameter that can bring additional relps to the Ewald sphere. At these wavelengths the observed spectrum shows extra peaks (Umweganregung) or dips (Aufhellung), colloquially called 'glitches' by X-ray spectroscopists. By analysing the passage of such relps through the Ewald sphere when varying its radius and the position of its centre, the relps can be identified, and the positions of the glitches can be calculated on the Braggangle and wavelength scales. Such an assignment is a necessary prerequisite for a reliable correction of the measured Compton profile for glitches.

In our calculation, the size of the diffracting domain of the relps is accounted for by varying the radius of the Ewald sphere from $0.999 r_{\mathrm{E}}^{*}$ to $1.001 r_{\mathrm{E}}^{*}$, and the result is shown in graph (b) of Fig. 4. Each linear chain of dots (interval of $0.01^{\circ}$ in $\theta_{\mathrm{B}}$ ) represents the passage of an extra relp through the shell around the Ewald sphere. Invisible in the graph are the extra relps (400) and (040), since they remain exactly on the sphere for all $\theta_{\mathrm{B}}$.

However, the percentage of incident and outgoing beams that are strictly parallel to the plane [1 10$]$ of the Rowland circle is differentially small. Namely, the beam hitting the analyser has a vertical divergence of $\pm 1.2^{\circ}$, and therefore the Ewald sphere possesses all tilts around the reciprocal lattice vector (440) within $\psi= \pm 1.2^{\circ}$ with respect to the plane [11̄0]. Therefore the glitches have been calculated also for $\psi=+1^{\circ}$ and $\psi=-1^{\circ}$ [graphs $(a)$ and $(c)$ in Fig. 4] in order to discriminate extra reflections whose $\theta_{\mathrm{B}}$ depends on $\psi$ only in second order from those that show first-order dependence on $\theta_{\mathrm{B}}$. The multiple diffraction with (400) and (040) is thus irrelevant,

(a)

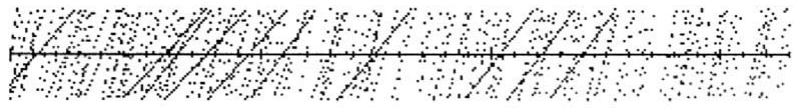

(b)

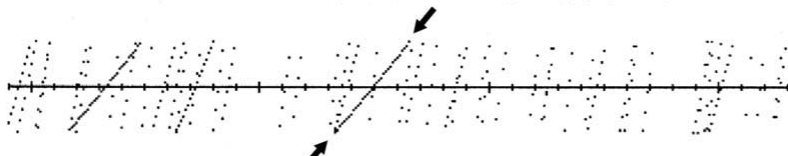

(c)

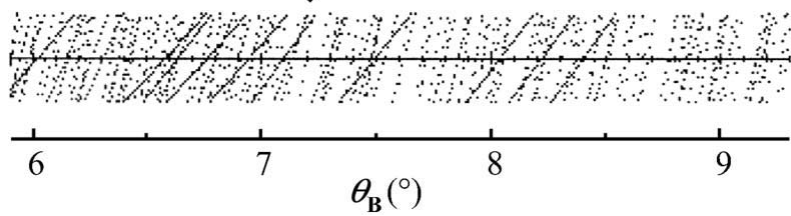

Figure 4

Angular positions of extra reflections $(b)$ for beams parallel to the plane of the Rowland circle $\left(\psi=0^{\circ}\right)$, and for beams inclined to that plane by $(a)$ $\psi=+1^{\circ}$ and $(c) \psi=-1^{\circ}$ around the (440) vector. The bands show the trajectories of reciprocal lattice points (relps) from $0.999 r_{\mathrm{E}}^{*}$ to $1.001 r_{\mathrm{E}}^{*}$ through the Ewald sphere of radius $r_{\mathrm{E}}^{*}$. The multiple reflection that gives rise to an observable glitch is marked by arrows in graph $(b)$ and can be found at almost the same $\theta_{\mathrm{B}}$ also in the graphs $(a)$ and $(c)$. since it is of the latter type, whereas the former type can yield a significant glitch by its integral over $\psi$ at almost constant $\theta_{\mathrm{B}}$.

The map of glitches shown in Fig. 4 presents only a finite fraction of extra reflections up to a limiting radius of $17 a^{*}$ in reciprocal space, whereas in total there are more than 16000 extra reflections in the scan range. An exact calculation of the integrated intensities of the extra reflections is not feasible in the case of a bent crystal, but fortunately most reflections come from distant relps, and are very weak. It is sufficient to identify the few reflections of relatively low order $(h k l)$ with second-order dependence on $\psi$. The only ones found are ( $3 \overline{3} 1)$ and (771), whose contribution [coinciding with those of some other high-order $(h k l)]$ is indicated in Fig. $4(b)$ by a pair of arrows, cuts the abscissa at $\theta_{\mathrm{B}}=\arctan (4 \sqrt{2} / 43) \simeq 7.494495^{\circ}$, and has already been observed by Suortti et al. (1999). The lines of dots for $\psi=+1^{\circ}$ (Fig. $4 a$ ) and $\psi=-1^{\circ}$ (Fig. 4c) are located at $\theta_{\mathrm{B}}=7.49306^{\circ}$, hardly different from the value at $\psi=$ 0 . These Bragg angles are absolute values that depend only on the cubic symmetry of the reciprocal lattice. Therefore we have used the glitch for the high-accuracy zero-point calibration of the experimental $\theta_{\mathrm{B}}$ scale for each crystallographic orientation of our samples separately.

With the primary energy and the scattering angle of the present experiment, that glitch is located in the momentum scale at $q=1.415 p_{0}$ with no counterpart at $q=-1.415 p_{0}$. Because of their completely different origin the glitches are not at symmetric positions with respect to $q=0$, so that glitchfree $J(|q|)$ can always be found.

The ratio $C(q)=J_{\text {uncorr }}(-q) / J_{\text {uncorr }}(+q)$, shown in Fig. 5, gives the analysers $q$-dependent quotient of its local quantum efficiency $\eta(q)$. The coincidence of the reflections (440), ( $\overline{3} \overline{3} 1)$, (771), $(\overline{10}, \overline{10}, 8),(14,14,8)$ etc. causes an $8 \%$ effect at $q=$ $+1.4 p_{0}$, which is about $1.2 \%$ of $J(0)$. The effects of the other glitches at $q=3.152,2.116$ and $3.127 p_{0}$ are much smaller,

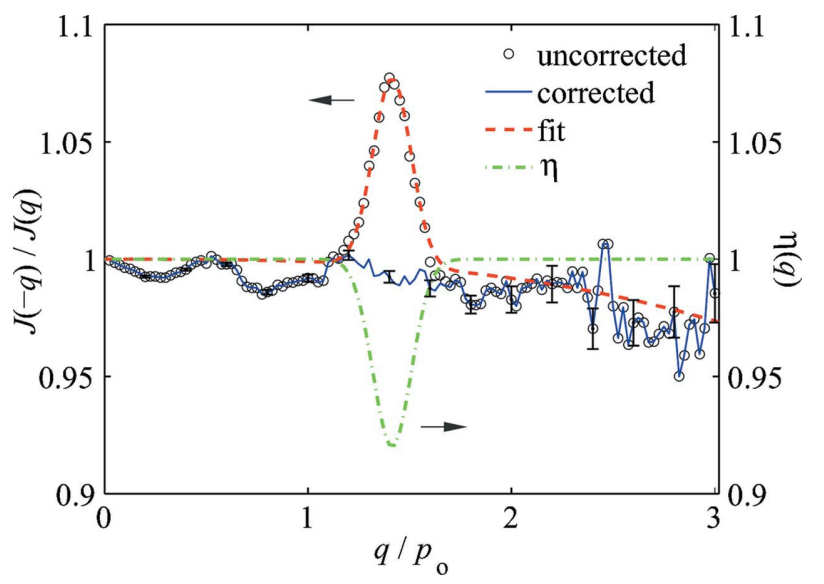

Figure 5

The $q$-dependent quotient $C(q)=J_{\text {uncorr }}(-q) / J_{\text {uncorr }}(+q)=\eta(-q) / \eta(+q)$ of the analysers local quantum efficiency $\eta(q)$. The local dip in $\eta(q)$ centred at $q=1.415 p_{0}$ is extracted by fitting a gross third-order polynomial plus a local Gaussian function to $C(q)$ and making use of the absence of a local glitch in $\eta(q)$ around $q=-1.415 p_{0}$. The dash-dotted line shows the resulting local $\eta(q)$ (right-hand scale), which $J_{\text {uncorr }}(q)$ is then corrected for. 
definitely less than $0.1 \%$ of $J(0)$ and undetectable in the counting statistics. The experimental Compton profiles, which had been corrected for other factors as described above, were divided locally in the range of the glitch by $\eta(q)$. It must be emphasized, however, that the asymmetry of the quantum efficiency $\eta(q)$ of the analyser could not have been calculated without the prior corrections for the deviations from IA, multiple scattering and the changing resolution function.

Glitches are unavoidable in all crystal spectrometers, although this fact is not always recognized or admitted. When the goal of Compton profile measurements is an accuracy of $0.1 \%$ of the peak value, a careful analysis and correction of the effects of glitches is essential. Probably the best approach to the problem is experimental, where scattering is measured with very high precision from a material that is known to have a smooth Compton profile that decreases monotonically with $q$. A polycrystalline sample of a free-electron metal, such as $\mathrm{Al}$, is a good choice.

\section{Results and discussion}

An example of the fully corrected Compton profile is shown in Fig. 6 , together with the asymmetry $J(+q)-J(-q)$ of the uncorrected profile. It is clear that an accuracy of $0.1 \%$ of the peak value would not have been reached without the detailed analysis described above. For a comparison, the differences $\Delta J(q)$ between a few crystallographic directions are shown in Fig. 7. The results for fully corrected and uncorrected profiles are almost identical, which demonstrates that the traditional use of directional differences in comparison with theory has been justified. The fully corrected Compton profiles are tabulated in Table 1.

When the fully corrected profiles are compared with the results of theory, the latter must be convoluted by the resolution function (or the experimental profile deconvoluted). The resolution function is very closely Gaussian, so that its Fourier transform is Gaussian, too. Because $B(s)$ is the Fourier

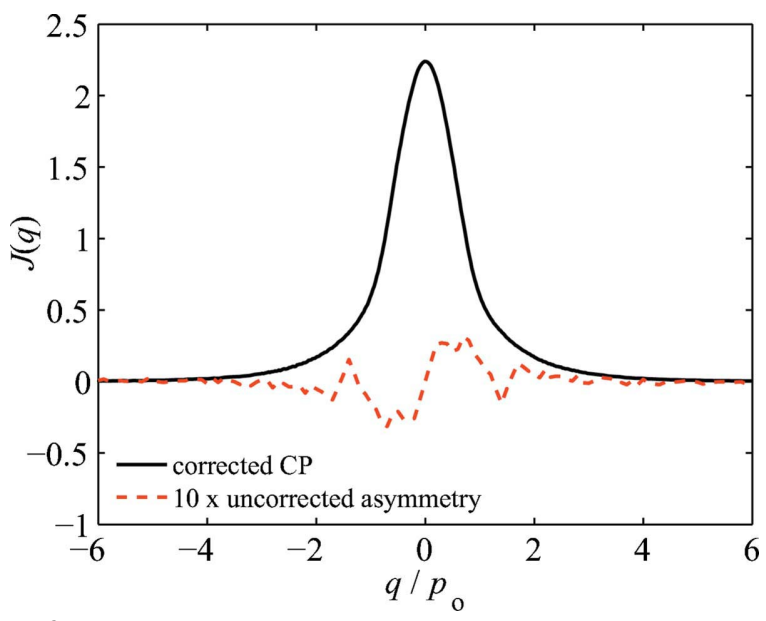

Figure 6

Fully corrected Compton profile in the [111] direction together with the magnified asymmetry $10 \times[J(+q)-J(-q)]$ of the uncorrected profile.

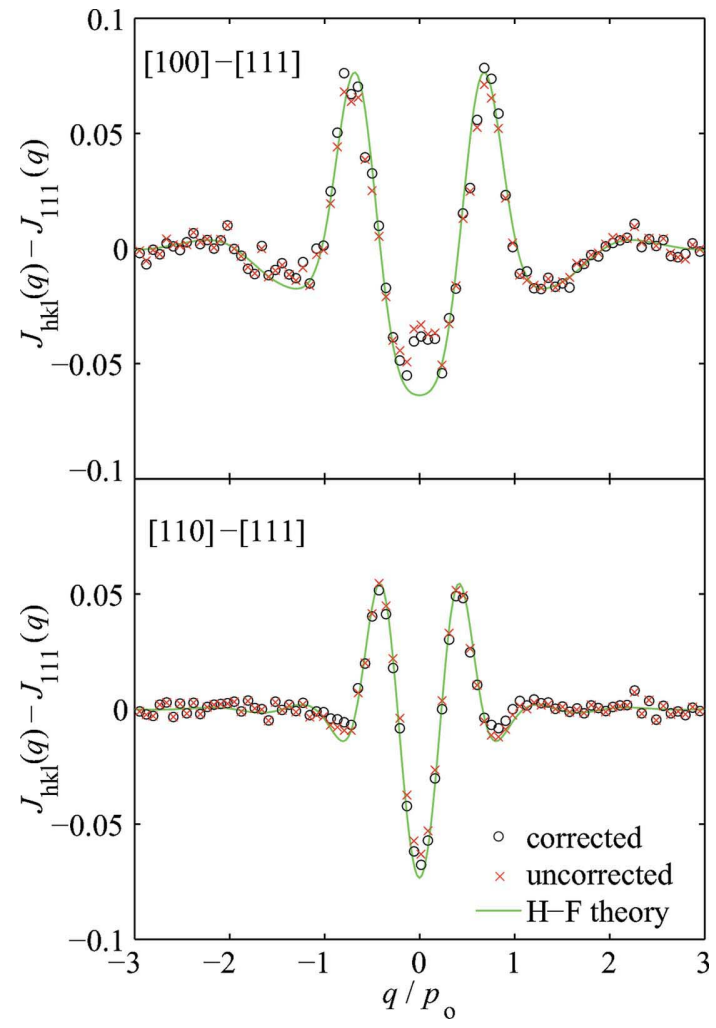

Figure 7

Differences between directional Compton profiles: $J_{100}-J_{111}$ and $J_{110}-$ $J_{111}$ for fully corrected profiles (open circles), uncorrected profiles (crosses) and theoretical calculations (full lines; Asthalter et al., 1992).

transform of $J(q)$, the effect of instrumental broadening is seen in $B(s)$ as a multiplicative Gaussian damping factor $\exp \left[-(s / S)^{2}\right]$, where $S=\left[4(\ln 2)^{1 / 2}\right] / \Delta q_{\mathrm{FWHM}}$. In the present case the average value is $S=15.86 a_{0}=8.39 \AA$. The theoretical $B(s)$ values were calculated in the directions where the DCPs were measured, and multiplied by individual damping factors instead of using the average $S$. The theoretical DCP was calculated by Fourier transforming the corresponding $B(s)$. The experimental $B(s)$ is obtained by transforming the experimental $J(q)$. This is an easy and transparent way to compare theory and experiment, either using $J(q)$ or $B(s)$. Experimental and theoretical reciprocal form factors in the principal crystallographic directions are shown in Fig. 8. Both the uncorrected and fully corrected experimental results are shown, and it is seen that the glitches cause unphysical oscillations in $B$ at high values of $s$.

Directional theoretical and experimental reciprocal form factors provide a starting point for reconstruction of the EMD. The three-dimensional function $B(\mathbf{s})$ is obtained by interpolation from the directional form factors, or it can be obtained by expansion into polyhedral harmonics $X_{\mathrm{L}}\left(\vartheta_{s}, \varphi_{s}\right)$ that have the correct Laue-group symmetry. The EMD $\varpi(\mathbf{p})$ is obtained by Fourier transforming $B(\mathbf{s})$, either numerically from the interpolated function, or by Hankel transformation of the radial factors $b_{\mathrm{L}}(s)$ of the polyhedral harmonics to $\varpi_{\mathrm{L}}(p)$. The results will be a part of a forthcoming article. 
Table 1

The fully corrected Compton profiles represented as the difference between each directional Compton profile and the spherically averaged Compton profile $\langle J(q)\rangle_{\Omega} / p_{0}^{-1}$.

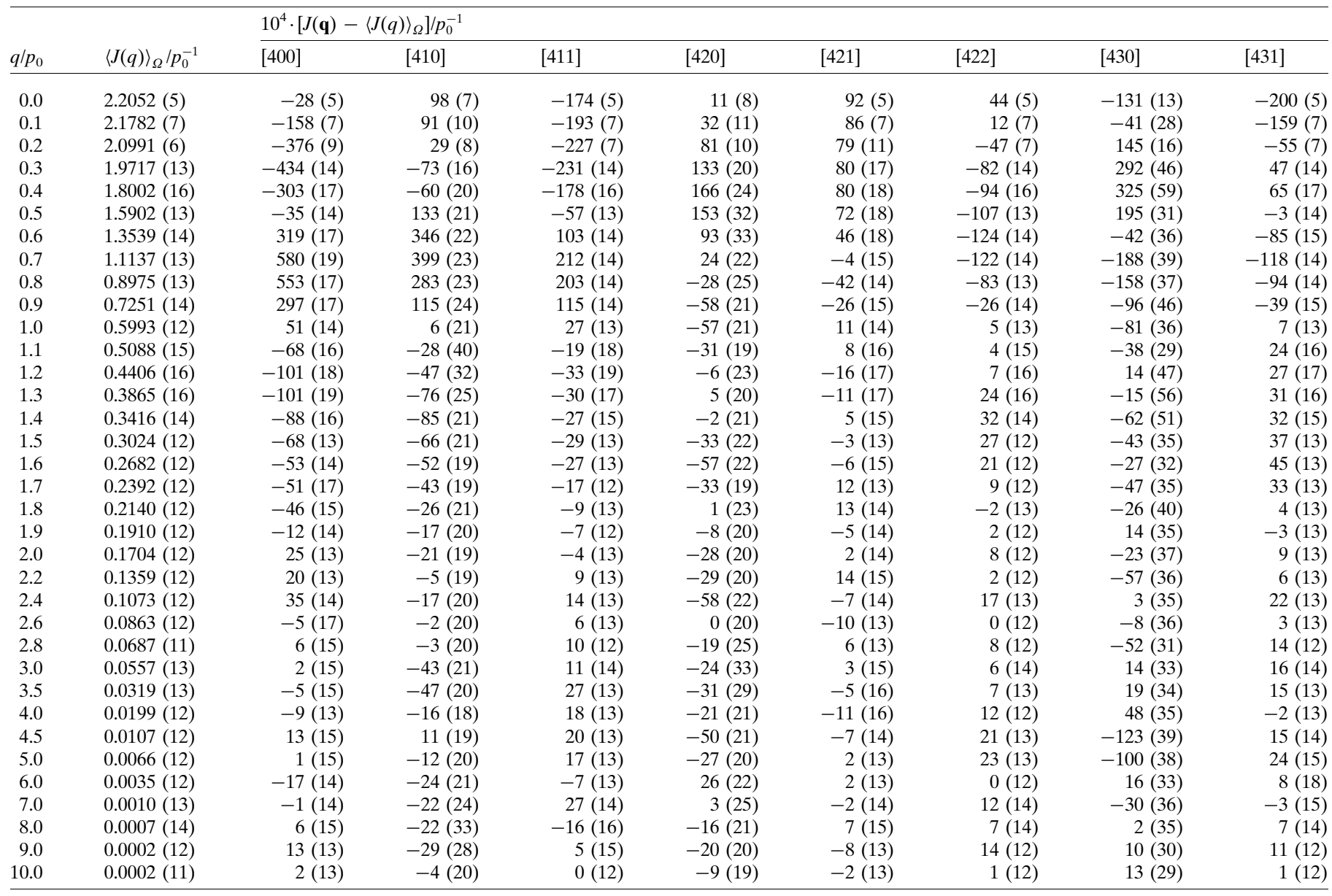

$10^{4} \cdot\left[J(\mathbf{q})-\langle J(q)\rangle_{\Omega}\right] / p_{0}^{-1}$

\begin{tabular}{|c|c|c|c|c|c|c|c|c|}
\hline$q / p_{0}$ & $\langle J(q)\rangle_{\Omega} / p_{0}^{-1}$ & [432] & [433] & [440] & [441] & {$[442]$} & {$[443]$} & {$[444]$} \\
\hline 0.0 & $2.2052(5)$ & $79(5)$ & $315(5)$ & $-323(5)$ & $-217(5)$ & $98(5)$ & $291(5)$ & $352(5)$ \\
\hline 0.1 & $2.1782(7)$ & $58(7)$ & $247(8)$ & $-231(7)$ & $-156(7)$ & $89(7)$ & $236(7)$ & $292(7)$ \\
\hline 0.2 & $2.0991(6)$ & $2(7)$ & $103(7)$ & $-16(8)$ & $-11(7)$ & $75(7)$ & $98(7)$ & $119(7)$ \\
\hline 0.3 & 1.9717 (13) & $-65(14)$ & $-25(14)$ & $184(15)$ & $116(14)$ & $53(14)$ & $-67(14)$ & $-105(14)$ \\
\hline 0.4 & $1.8002(16)$ & $-117(17)$ & $-120(16)$ & 234 (17) & $126(16)$ & $-19(16)$ & $-200(16)$ & $-261(16)$ \\
\hline 0.5 & 1.5902 (13) & $-151(14)$ & $-191(14)$ & 101 (14) & 12 (13) & $-124(13)$ & $-244(13)$ & $-288(13)$ \\
\hline 0.6 & $1.3539(14)$ & $-162(15)$ & $-217(14)$ & $-102(14)$ & $-132(14)$ & $-185(14)$ & $-205(14)$ & $-231(15)$ \\
\hline 0.7 & 1.1137 (13) & $-127(14)$ & $-181(14)$ & $-210(14)$ & $-198(14)$ & $-172(14)$ & $-149(14)$ & $-157(13)$ \\
\hline 0.8 & 0.8975 (13) & $-58(14)$ & $-109(14)$ & $-170(13)$ & $-152(14)$ & $-122(14)$ & $-106(14)$ & $-85(14)$ \\
\hline 0.9 & $0.7251(14)$ & $-7(15)$ & $-43(14)$ & $-66(14)$ & $-53(14)$ & $-62(15)$ & $-46(15)$ & $-24(15)$ \\
\hline 1.0 & 0.5993 (12) & $12(13)$ & $-10(13)$ & $8(13)$ & 15 (13) & $-5(13)$ & 13 (13) & 9 (12) \\
\hline 1.1 & $0.5088(15)$ & $30(15)$ & $-4(16)$ & $39(15)$ & $29(16)$ & $25(16)$ & $29(15)$ & $19(15)$ \\
\hline 1.2 & $0.4406(16)$ & 47 (16) & 2 (19) & 47 (16) & 31 (18) & $29(17)$ & $25(16)$ & $26(17)$ \\
\hline 1.3 & $0.3865(16)$ & $40(16)$ & 18 (19) & 46 (16) & 42 (18) & 30 (18) & 43 (16) & $34(16)$ \\
\hline 1.4 & $0.3416(14)$ & $29(15)$ & 27 (16) & $42(15)$ & 49 (16) & $38(15)$ & $61(15)$ & 39 (15) \\
\hline 1.5 & 0.3024 (12) & 38 (13) & $27(15)$ & $40(12)$ & 43 (14) & 41 (13) & $51(12)$ & $38(12)$ \\
\hline 1.6 & 0.2682 (12) & 44 (12) & $24(13)$ & 33 (12) & 35 (12) & 32 (12) & $33(12)$ & $31(12)$ \\
\hline 1.7 & 0.2392 (12) & 31 (13) & 17 (12) & $21(12)$ & $23(12)$ & $16(13)$ & $26(12)$ & $22(12)$ \\
\hline 1.8 & $0.2140(12)$ & $20(13)$ & $5(14)$ & $13(13)$ & $12(13)$ & $6(13)$ & $18(13)$ & $18(13)$ \\
\hline 1.9 & 0.1910 (12) & $18(13)$ & $2(12)$ & $10(12)$ & 17 (13) & $8(13)$ & $8(12)$ & 15 (12) \\
\hline 2.0 & 0.1704 (12) & $10(13)$ & $9(13)$ & 9 (13) & 31 (13) & $9(13)$ & $5(12)$ & $6(12)$ \\
\hline 2.2 & 0.1359 (12) & $14(13)$ & $-2(13)$ & 17 (13) & $14(13)$ & $-17(13)$ & $12(12)$ & $-6(12)$ \\
\hline 2.4 & 0.1073 (12) & $9(13)$ & $-3(13)$ & 7 (13) & $0(13)$ & 14 (13) & $-2(13)$ & 7 (12) \\
\hline 2.6 & 0.0863 (12) & $10(13)$ & 0 (13) & $-2(13)$ & $5(13)$ & $-4(13)$ & 11 (13) & $3(12)$ \\
\hline 2.8 & 0.0687 (11) & $-6(13)$ & $5(13)$ & $4(12)$ & 7 (14) & $16(14)$ & $-2(12)$ & $16(12)$ \\
\hline 3.0 & 0.0557 (13) & $16(14)$ & $8(15)$ & $1(14)$ & $-1(14)$ & $-1(14)$ & 7 (14) & $3(14)$ \\
\hline 3.5 & $0.0319(13)$ & $2(13)$ & $5(13)$ & $6(14)$ & $11(13)$ & 19 (13) & $14(13)$ & $20(13)$ \\
\hline 4.0 & 0.0199 (12) & $-3(13)$ & 0 (12) & $8(12)$ & $10(13)$ & $-9(14)$ & $5(12)$ & $5(12)$ \\
\hline
\end{tabular}


Table 1 (continued)

\begin{tabular}{|c|c|c|c|c|c|c|c|c|}
\hline \multirow[b]{2}{*}{$q / p_{0}$} & \multirow[b]{2}{*}{$\langle J(q)\rangle_{\Omega} / p_{0}^{-1}$} & \multicolumn{7}{|c|}{$10^{4} \cdot\left[J(\mathbf{q})-\langle J(q)\rangle_{\Omega}\right] / p_{0}^{-1}$} \\
\hline & & [432] & [433] & [440] & [441] & [442] & [443] & [444] \\
\hline 4.5 & $0.0107(12)$ & $21(14)$ & $29(13)$ & $16(13)$ & $20(13)$ & $17(15)$ & $20(13)$ & $20(13)$ \\
\hline 7.0 & 0.0010 (13) & $-4(14)$ & $12(14)$ & 4 (14) & $10(14)$ & $2(14)$ & $2(14)$ & $12(13)$ \\
\hline 8.0 & 0.0007 (14) & $16(14)$ & $6(14)$ & $5(14)$ & $8(16)$ & $-4(14)$ & $8(14)$ & $1(14)$ \\
\hline 9.0 & 0.0002 (12) & $8(13)$ & $19(12)$ & $3(12)$ & $4(16)$ & $4(13)$ & $9(12)$ & $14(12)$ \\
\hline
\end{tabular}

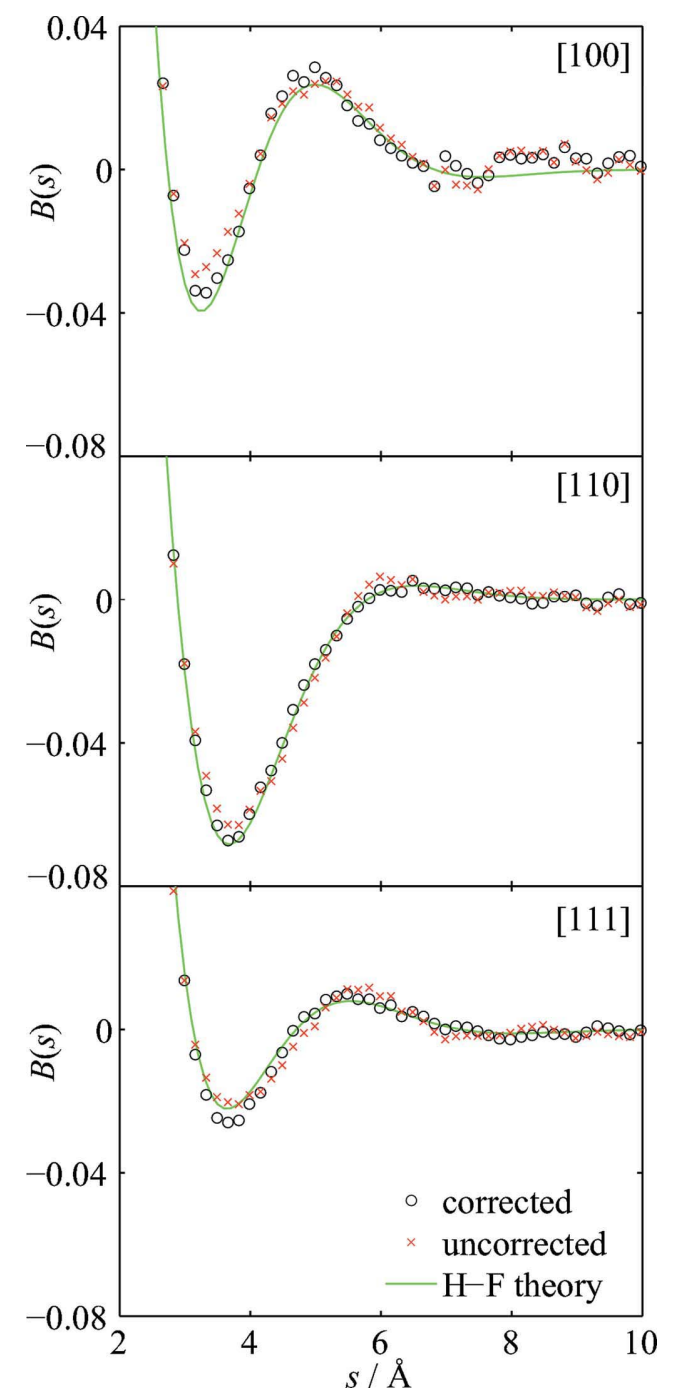

Figure 8

Experimental and theoretical reciprocal form factors, which have been corrected for instrumental broadening, along radial lines in the [100], [110] and [111] directions. Statistical error bars are of the same size as the plotting symbols.

\section{Conclusions}

It has been demonstrated that highly accurate experimental directional Compton profiles can be extracted from the inelastic scattering spectrum recorded by a scanning crystal spectrometer. An accuracy of $0.1 \%$ of the peak value is reached, because the background is low and can be deter- mined separately and subtracted, the reflectivity of the analyser crystal can be calculated, and the resolution function can be measured independently. It is shown that the corrections for the deviation from the IA, multiple scattering and a non-constant resolution function must be calculated individually, and that glitches must be identified and their effects removed.

The purpose of this work is to give a detailed account of the procedures required for reaching an accuracy of $0.1 \%$ at the Compton peak. Such accuracy is needed when discussing effects that are still challenges to theory. In the case of $\mathrm{LiH}$ the electron wavefunction can be calculated at the Hartree-Fock level, so that the differences between theory and experiment should arise from electron-electron correlation only. There are several theoretical approaches, and results from highly accurate Compton profile measurements are capable of providing stringent tests for theory. A full account of the experimental Compton profiles and reciprocal form factors of $\mathrm{LiH}$ will be presented in subsequent publications where comparisons with earlier experimental work and theoretical calculations will also be given.

The authors thank T. Buslaps, N. Hiraoka and H. Müller for their help in the experiments, and the ESRF for the beam time. The financial support of The Academy of Finland and the Fonds der Chemischen Industrie is gratefully acknowledged.

\section{References}

Asthalter, T. \& Weyrich, W. (1993). Z. Naturforsch. Teil A, 48, $303-$ 309.

Asthalter, T. \& Weyrich, W. (1997). Ber. Bunsenges. Phys. Chem. 101, $11-22$.

Asthalter, T., Weyrich, W., Harker, A. H., Kunz, A. B., Orlando, R. \& Pisani, C. (1992). Solid State Commun. 83, 725-730.

Berthold, A., Mourikis, S., Schmitz, J. R., Schülke, W. \& SchulteSchrepping, H. (1992). Nucl. Instrum. Methods Phys. Res. A, 317, 373-382.

Boldrini, B. \& Weyrich, W. (2010). In preparation.

Bräuchler, M., Lunell, S., Olovsson, I. \& Weyrich, W. (1989). Int. J. Quantum Chem. 35, 895-917.

Cooper, M. J., Mijnarends, P. E., Shiotani, N., Sakai, N. \& Bansil, A. (2004). Editors. X-ray Compton Scattering. Oxford University Press.

Dugdale, S. B. \& Jarlborg, T. (1998). Solid State Commun. 105, $283-$ 287.

DuMond, J. W. M. (1947). Rev. Sci. Instrum. 18, 626-638. 
DuMond, J. W. M. \& Kirkpatrick, H. A. (1930). Rev. Sci. Instrum. 1, 88-105.

Eisenberger, P. \& Platzman, P. M. (1970). Phys. Rev. A, 2, 415-423.

Erola, E., Eteläniemi, V., Suortti, P., Pattison, P. \& Thomlinson, W. (1990). J. Appl. Cryst. 23, 35-42.

Gillet, J. M., Becker, P. J. \& Loupias, G. (1995). Acta Cryst. A51, 405413.

Fajardo, P., Honkimäki, V., Buslaps, T. \& Suortti, P. (1998). Nucl. Instrum. Methods Phys. Res. B, 134, 337-345.

Hiraoka, N., Buslaps, T., Honkimäki, V., Guyot, H. \& Schlenker, C. (2005a). Phys. Rev. B, 71, 125417.

Hiraoka, N., Buslaps, T., Honkimäki, V. \& Suortti, P. (2005b). J. Synchrotron Rad. 12, 670-674.

Hiraoka, N., Itou, M., Ohata, T., Mizumaki, M., Sakurai, Y. \& Sakai, N. (2001). J. Synchrotron Rad. 8, 26-32.

Holm, P. (1988). Phys. Rev. A, 37, 3706-3719.

Holm, P. \& Ribberfors, R. (1989). Phys. Rev. A, 40, 6251-6259.

Huotari, S., Hämäläinen, K., Manninen, S., Issolah, A. \& Marangolo, M. (2001). J. Phys. Chem. Solids, 62, 2205-2213.

Huotari, S., Hämäläinen, K., Manninen, S., Kaprzyk, S., Bansil, A., Caliebe, W., Buslaps, T., Honkimäki, V. \& Suortti, P. (2000). Phys. Rev. B, 62, 7956-7963.

Huotari, S., Hämäläinen, K., Manninen, S., Sternemann, C., Kaprolat, A., Schülke, W. \& Buslaps, T. (2002). Phys. Rev. B, 66, 085104.

Huotari, S., Sternemann, C., Volmer, M., Soininen, J. A., Monaco, G. \& Schülke, W. (2007). Phys. Rev. B, 76, 235106.

Issolah, A., Garreau, Y., Levy, B. \& Loupias, G. (1991). Phys. Rev. B, 44, 11029-11034.

Janis, W. J., Kaijser, P., Smith, V. H. Jr \& Whangbo, M.-H. (1978). Mol. Phys. 35, 1237-1245.

Laundy, D. \& Collins, S. (2003). J. Synchrotron Rad. 10, 214-218.

Loupias, G. \& Mergy, J. (1980). Unpublished Report, cited by J.-M. Gillet et al. (1995).

Loupias, G. \& Petiau, J. (1980). J. Phys. (Paris), 41, 265-271.

Manninen, S., Honkimäki, V., Hämäläinen, K., Laukkanen, J., Blaas, C., Redinger, J., McCarthy, J. \& Suortti, P. (1996). Phys. Rev. B, 53, 7714-7720.

Nyanda, A. M. \& Williams, B. G. (1979). Philos. Mag. B, 40, 97-104.
Nygård, K., Hakala, M., Manninen, S., Itou, M., Sakurai, Y. \& Hämäläinen, K. (2007). Phys. Rev. Lett. 99, 197401.

Pattison, P. \& Weyrich, W. (1979). J. Phys. Chem. Solids, 40, 213-222.

Sakurai, Y., Ito, M., Urai, T., Tanaka, Y., Sakai, N., Iwazumi, T., Kawata, H., Ando, M. \& Shiotani, N. (1992). Rev. Sci. Instrum. 63, 1190-1193.

Sakurai, Y., Tanaka, Y., Bansil, A., Kaprzyk, S., Stewart, A. T., Nagashima, Y., Hyodo, T., Nanao, S., Kawata, H. \& Shiotani, N. (1995). Phys. Rev. Lett. 74, 2252-2255.

Schülke, W., Stutz, G., Wohlert, F. \& Kaprolat, A. (1996). Phys. Rev. $B, \mathbf{5 4}, 14381-14395$.

Shiotani, N., Sakai, N., Itoh, F., Sakurai, M., Kawata, H., Amemiya, Y. \& Ando, M. (1989). Nucl. Instrum. Methods Phys. Res. A, 275, $447-$ 452.

Shukla, A., Barbiellini, B., Erb, A., Manuel, A., Buslaps, T., Honkimäki, V. \& Suortti, P. (1999). Phys. Rev. B, 59, 12127-12131.

Soininen, J. A., Hämäläinen, K. \& Manninen, S. (2001). Phys. Rev. B, 64, 125116.

Sternemann, C., Buslaps, T., Shukla, A., Suortti, P., Döring, G. \& Schülke, W. (2001). Phys. Rev. B, 63, 094301.

Sternemann, C., Hämäläinen, K., Kaprolat, A., Soininen, A., Döring, G., Kao, C.-C., Manninen, S. \& Schülke, W. (2000). Phys. Rev. B, 62, R7687-R7690.

Suortti, P., Buslaps, T., DiMichiel, M., Honkimäki, V., Lienert, U., McCarthy, J. E., Merino, J. M. \& Shukla, A. (2001). Nucl. Instrum. Methods Phys. Res. A, 467-468, 1541-1544.

Suortti, P., Buslaps, T., Fajardo, P., Honkimäki, V., Kretzschmer, M., Lienert, U., McCarthy, J. E., Renier, M., Shukla, A., Tschentscher, T. \& Meinander, T. (1999). J. Synchrotron Rad. 6, 69-80.

Tanaka, Y., Sakurai, Y., Stewart, A. T., Shiotani, N., Mijnarends, P. E., Kaprzyk, S. \& Bansil, A. (2001). Phys. Rev. B, 63, 045120.

Weyrich, W. (1978). Habilitation thesis, Technical University of Darmstadt, Germany.

Weyrich, W. (1979). Ber. Bunsenges. Phys. Chem. 83, 797-807.

Weyrich, W. (1996). Quantum-Mechanical ab initio Calculations of the Properties of Crystalline Materials, edited by C. Pisani, pp. 245-272. Berlin: Springer.

Weyrich, W. (2010). In preparation. 